Table 3. Interatomic distances and bond angle

$$
\begin{array}{lll}
\mathrm{Cu}-\mathrm{O}_{\mathrm{I}}=2 \cdot 00 \AA & \mathrm{O}_{\mathrm{IV}}-\mathrm{O}_{\mathrm{II}}=2 \cdot 82 \AA & \mathrm{C}-\mathrm{O}_{\mathrm{I}}=1 \cdot 26 \AA \\
\mathrm{Cu}-\mathrm{O}_{\mathrm{II}}=2 \cdot 01 & \mathrm{O}_{\mathrm{IV}}-\mathrm{O}_{\mathrm{III}}^{\prime}=2 \cdot 78 & \mathrm{C}-\mathrm{O}_{\mathrm{II}}=1 \cdot 25 \\
\mathrm{Cu}-\mathrm{O}_{\mathrm{III}}=2 \cdot 36 & \mathrm{O}_{\mathrm{IV}}-\mathrm{O}_{\mathrm{III}}=2 \cdot 74 & \\
& \angle \mathrm{O}-\mathrm{C}-\mathrm{O}=120^{\circ} &
\end{array}
$$

in Table 3. Each copper atom is surrounded by four oxygen atoms of four different formate ions in an approximately square configuration with $\mathrm{Cu}-\mathrm{O}$ distances $2.00 \AA$ and $2.01 \AA$ and, above and below this plane, by two equivalent water molecules $\left(\mathrm{O}_{\text {III }}\right)$ with a far longer $\mathrm{Cu}-\mathrm{O}$ distance $(2 \cdot 36 \AA)$. Such facts suggest that there is a considerable difference between the nature of these $\mathrm{Cu}-\mathrm{O}$ bindings, and this also accounts for the strong pleochroism. Another water molecule $\left(\mathrm{O}_{\text {IV }}\right)$ is not linked to the copper atom, but to one $\mathrm{O}_{\mathrm{II}}$ and two $\mathrm{O}_{\mathrm{III}}$ 's with hydrogen-bond distances $2 \cdot 82$, 2.74 and $2.78 \AA$. The formate ion has a nearly symmetrical configuration with the $\mathrm{C}-\mathrm{O}$ distances 1.25 and $1.26 \AA$ and the bond angle $\angle \mathrm{O}-\mathrm{C}-\mathrm{O}$ about $120^{\circ}$.
As shown in Fig. 1, there is formed a distinct layer structure in which one formate ion is coordinated to two copper atoms. Such layers are parallel to (001) and both kinds of water molecules are sandwiched between these layers.

Owing to this predominant layer structure, this crystal shows highly perfect cleavage parallel to (001) and also large anisotropy in its dielectric behaviour and paramagnetic properties. Besides these, the very efflorescent nature of this tetrahydrate may be explained reasonably on the basis of this crystal structure.

The authors would like to thank Prof. I. Nitta for his interest and encouragement.

\section{References}

Cochran, W. (1951). Acta Cryst. 4, 81.

KabraJI, K. J. (1931). Indian J. Phys. 6, 81 (Abstract in Strukturbericht, 2, 843).

Acta Cryst. (1954). 7, 483

\title{
Generalisation du Principe de Focalisation de Bragg-de Broglie
}

\author{
Par A. J. Rost \\ Laboratoire de Minéralogie, Sorbonne, Paris, France
}

(Reçu le 5 janvier 1954)

\begin{abstract}
On the basis of the Bragg-de Broglie principle of focusing the author has studied the focusing of a pencil of X-rays diffracted by lattice planes inclined to the reflecting face of the crystal. A discussion is given of applications to emission spectroscopy and to the formation of radiographic images of thin crystal plates.
\end{abstract}

\section{Introduction}

Bragg \& Bragg (1914) et de Broglie (1914) ont montré que les faisceaux diffractés par les plans réticulaires parallèles à la surface plane d'une lame monocristalline tournant autour d'une droite de sa face, sont focalisés sur un cercle, section droite du cylindre de révolution, contenant la source ponctuelle de rayons $\mathrm{X}$ et ayant pour axe, l'axe de rotation du cristal.

D'autre part, Tellez-Plasencia (1943) a montré que d'autres conditions de focalisation peuvent être obtenues si la source et le film sont animés de mouvements convenables.

Dans cette note, on poursuit, sur les bases expérimentales de Bragg-de Broglie, l'étude géométrique des faisceaux diffractés par les plans réticulaires inclinés sur la face de réflexion; on envisage le cas des plans dont l'intersection avec la face de réflexion est parallèle à l'axe de rotation et on recherche les lieux de focalisation.

\section{Schéma expérimental}

Soient (Fig. 1(a) et $(b)$ ):

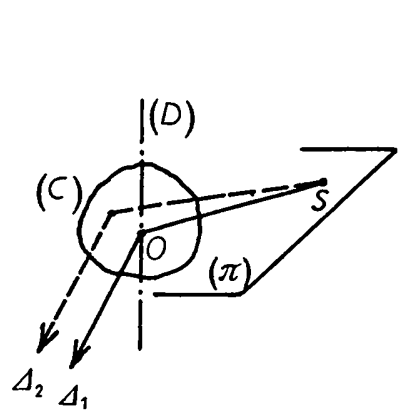

(a)

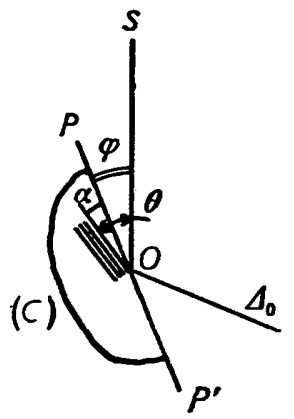

(b)
Fig. 1. Schéma expérimental. $S$, source de rayons $\mathrm{X}$; $(D)$ axe de rotation du cristal; $(\Delta)$ direction des faisceaux diffractés. (a) Cas général. (b) Cas des plans réticulaires ayant (D) comme axe de zone. Réflexion du rayon $S O$ dans le plan $(\pi)$ perpendiculaire à $(D)$. 
$(\pi)$, le plan de référence,

$S$, une source ponctuelle de rayons X monochromatiques dans le plan $(\pi)$,

$(C)$, un monocristal dont une face plane $P P^{\prime}$ est perpendiculaire au plan $(\pi)$,

$(D)$, l'axe de rotation du cristal contenu dans la face réfléchissante et perpendiculaire au plan $(\pi)$,

$O$, l'intersection du plan $(\pi)$ et de la droite $(D)$,

$\left(\Delta_{n}\right)$, la direction des faisceaux diffractés,

$\alpha$, l'angle d'inclinaison des plans réflecteurs sur la face de réflexion,

$\varphi$, l'angle d'incidence de la direction $S O$ sur la face de réflexion,

$\theta$, l'angle de Bragg,

$(\Sigma)$, le cercle de focalisation de Seeman-Bohlin dans le plan $(\pi)$ passant par $S$ et tangent à la surface de réflexion (Figs. 2-6).

Pour une position déterminée du cristal (Fig. 1 $(a)$ ) une famille de plans réticulaires $h k l$ ne réfléchit un des rayons issus de la source que si la loi de Bragg est vérifiée.

Supposons que dans cette position, le rayon $S O$ soit réfléchi dans la direction $\Delta_{1}$ pour une famille de plans réticulaires d'indice $h_{0} k_{0} l_{0}$.

Après une rotation $\varepsilon$ du cristal autour de la droite $(D)$, cette même famille de plans $h_{0} k_{0} l_{0}$, réfléchira un autre rayon incident issu de $S$, dans une direction $\Delta_{2}$ à condition que la loi de Bragg soit à nouveau vérifiée.

Si le cristal tourne autour de $(D)$ dans un domaine où la loi de Bragg est toujours vérifiée pour les plans $h_{0} k_{0} l_{0}$, le faisceau de rayons incidents sera diffracté suivant une famille de directions $\Delta_{1}, \Delta_{2}, \ldots, \Delta_{n}$.

C'est le faisceau de directions $\Delta_{n}$, que l'on va étudier pour les familles de plans dont la trace sur la face de réflexion est parallèle à l'axe de rotation.

\section{Etude géométrique des rayons diffractés par une famille de plans réticulaires ayant $(D)$, axe de rotation du cristal, comme axe de zone}

Considérons parmi les plans réflecteurs qui ont cette droite $(D)$ pour axe de zone, les plans d'intervalle $d_{h k l}$ qui forment avec la face $P P^{\prime}$ un angle $\alpha$ et supposons le cristal orienté de telle façon que cette famille de plans $h k l$, réfléchisse le rayon incident $S O$.

Après une rọtation $\varepsilon$ du cristal (Fig. 2), c'est le rayon $S I$, tel que l'on ait $\angle S I P_{1}=\angle S O P=\theta-\alpha$, qui est réfléchi.

Les deux rayons diffractés, ou plus exactement leur direction, se coupent en un point $F$ dont on se propose de définir la position.

Plusieurs cas sont à envisager; on peut avoir:

$\alpha<\theta$ avec $\varphi=\theta-\alpha$ (cas de la réflexion,

$\alpha<\theta$ avec $\varphi=\theta+\alpha\} \quad$ Figs. 3 et 4).

$\alpha>\theta$ avec $\varphi=\alpha-\theta$ ) (cas de la transmission,

$\alpha>\theta$ avec $\varphi=\alpha+\theta\} \quad$ Figs. 5 et 6 ).

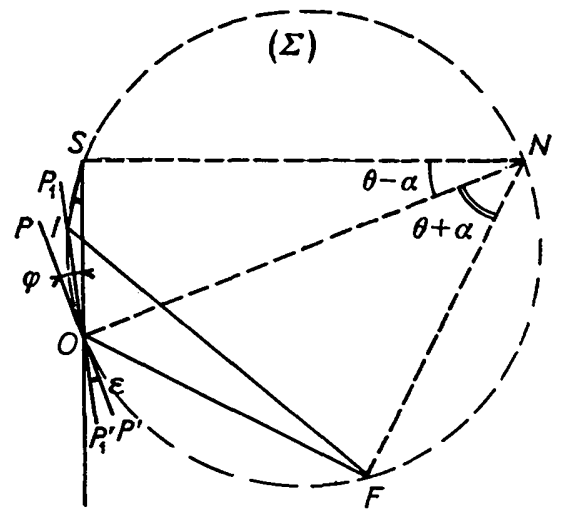

Fig. 2. Construction des rayons diffractés par des plans réticulaires inclinés sur la face de réflexion, après une rotation $\varepsilon$ du cristal. $P P^{\prime}$, position de la face de réflexion du cristal quand le ravon $S O$ est réfléchi; $(\Sigma)$, cercle de focalisation passant par $S$ et tangent en $O$ à $P P^{\prime} ; O N$, normale à $P P^{\prime}$.

(1) Cas de la réflexion

$$
\alpha<\theta \text { avec } \varphi=\theta-\alpha .
$$

Soit (Figs. 2 et 3) $P_{1} P_{1}^{\prime}$ la position de la face du

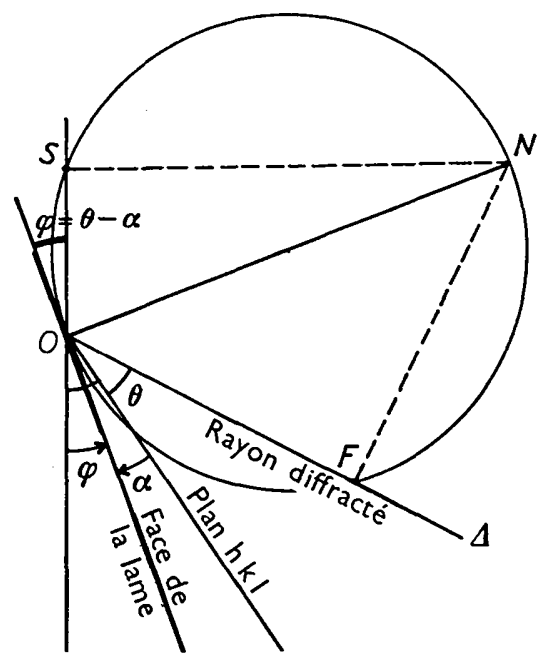

Fig. 3. Cas de la réflexion $\alpha<\theta$, avec $\varphi=\theta-\alpha$.

cristal après la rotation $\varepsilon$ et $I$ l'intersection de $(\Sigma)$ avec $P_{1} P_{1}^{\prime}$ :

$$
\angle S I P_{1}=\angle S O P=\varphi=\theta-\alpha .
$$

Les deux rayons diffractés se coupent sur le cercle $(\Sigma)$ puisque:

$$
\angle S I F=\angle S O F=\pi-2 \theta .
$$

Il suffit de considérer les triangles rectangles $O N S$ et $O N F$ pour trouver, en appelant $O S=l$, constante de l'appareil:

$$
O F=l \frac{\sin (\theta+\alpha)}{\sin (\theta-\alpha)} .
$$

$$
\alpha<\theta \quad \text { avec } \varphi=\theta+\alpha \quad \text { (Fig. 4). }
$$




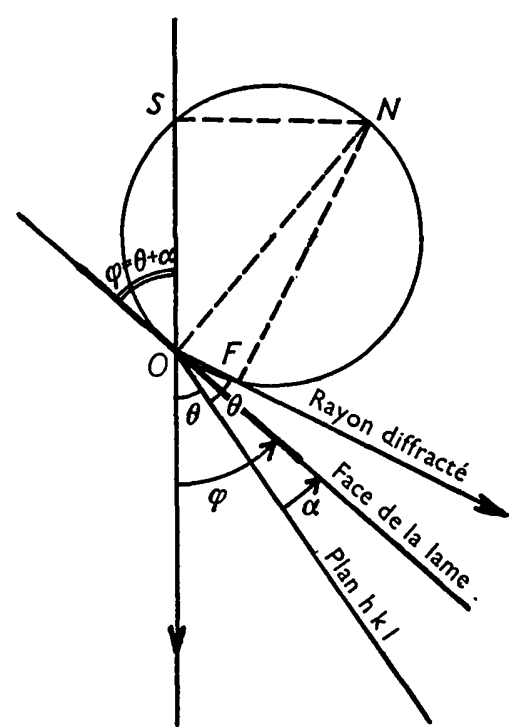

Fig. 4. Cas de la réflexion $\alpha<\theta$, avec $\varphi=\theta+x$.

On trouve de la même façon:

$$
O F=l \frac{\sin (\theta-x)}{\sin (\theta+x)} .
$$

(2) Cas de la transmission
(a)
$x>\theta$ avec $\varphi=x-\theta$
(Fig. 5).

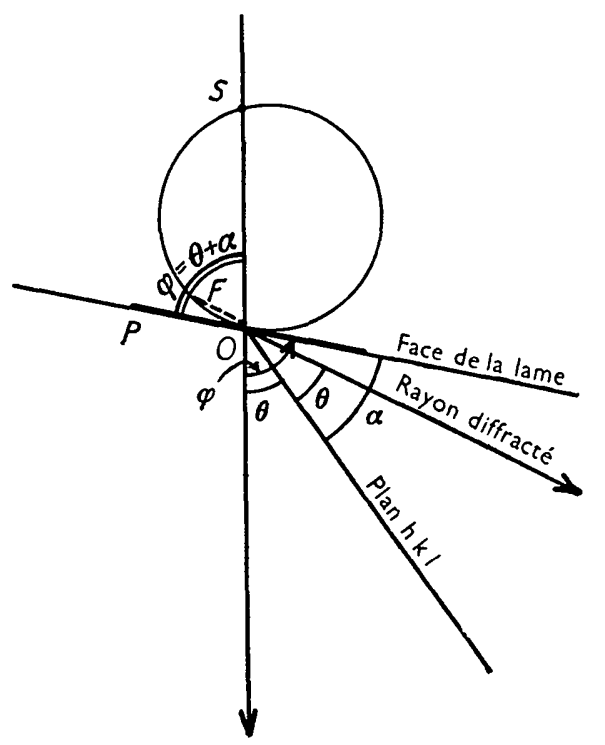

Fig. 5. Cas de la transmission $x>\theta$, avec $\varphi=\theta+x$.

On trouve:

$$
O F=l \frac{\sin (\alpha+\theta)}{\sin (\alpha-\theta)} .
$$

Mais $F$ est un foyer virtuel et l'on reçoit des faisceaux réfléchis divergents.

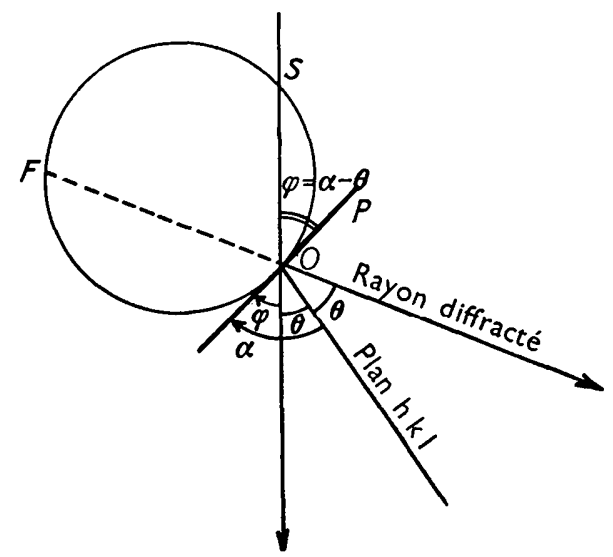

Fig. 6. Cas de la transmission $\alpha>\theta$, avec $\varphi=\alpha-\theta$.

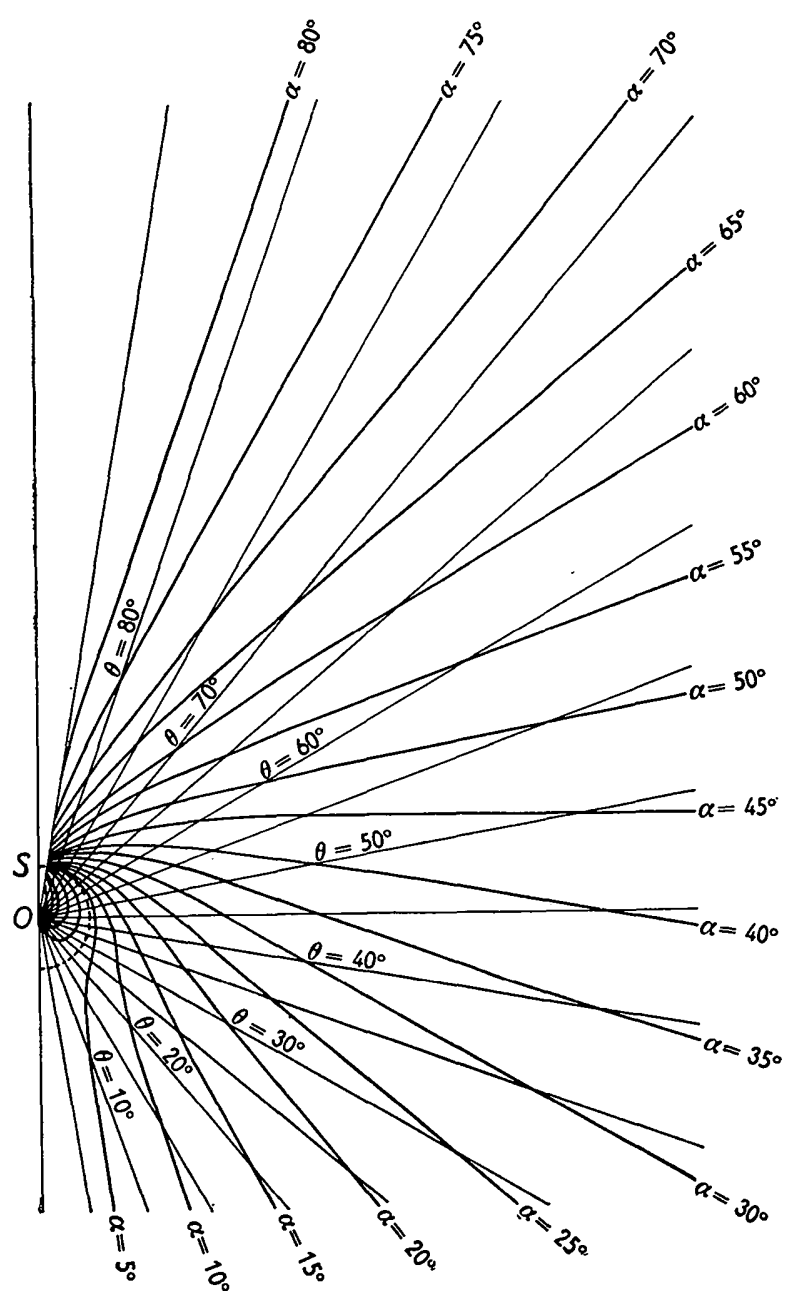

Fig. 7. Lieux de focalisation des faisceaux diffractés sous différents angles $\theta$ par les plans réticulaires inclinés d'un angle $\alpha$ sur la face de réflexion du cristal analyseur (schéma Fig. $1(b)$ ). Les courbes ont été calculées pour des angles $\alpha$ de 5 en $5^{\circ}$. 
(b) $\alpha>\theta$ avec $\varphi=\alpha+\theta \quad$ (Fig. 6).

On trouve:

$$
O F=l \frac{\sin (\alpha-\theta)}{\sin (\alpha+\theta)} \text {. }
$$

$F$ est également un foyer virtuel.

\section{Lieu des points de focalisation en fonction de $\boldsymbol{\theta}$}

Le système est déterminé quand on a les valeurs de $d_{h k l}$ intervalles réticulaires des plans réflecteurs, leur inclinaison sur le plan de la lame $(\alpha)$ et l'angle de la direction $S O$ avec la face de la lame $(\varphi)$; remarquons que les deux valeurs possibles de $\varphi(\theta \pm \alpha)$ équivalent à deux positions de la lame cristalline qui se déduisent l'une de l'autre par une rotation dans son plan de $180^{\circ}$ autour de la normale $O N$.

Si l'on éclaire la lame avec une source polychromatique divergente de rayons $\mathrm{X}$ on trouvera donc pour chaque longueur d'onde du faisceau incident, c'est-àdire, pour une série de valeurs de $\theta$, une série de points de focalisation $F$ définis par les relations (1) et (2).

L'abaque de la Fig. 7 représente le lieu de ces points $F$ pour différentes valeurs de $\alpha$ de 5 en $5^{\circ}$, et pour toutes les valeurs de $\theta$ compatibles $(\theta>\alpha$ pour le cas de la réflexion; $\theta<\alpha$ pour le cas de la transmission). Un tableau de valeurs calculées pour la construction de l'abaque peut être obtenu en s'adressant à l'auteur.

\section{Remarques}

1. Toutes ces courbes ont un point commun; la source $S$. (Quand $\theta=\pi / 2, O F=l$, quel que soit $\alpha$.)

2. Quand $\alpha$ est nul, la courbe est un cercle de centre $O$ et de rayon $O S ;(O F=l$ quel que soit $\theta)$; c'est le cas classique de Bragg-de Broglie: le cristal est placé au centre d'une chambre cylindrique, la source ponctuelle monochromatique divergente, à l'entrée de la chambre sur une génératrice du film, dans le plan équatorial; les plans réflecteurs parallèles à la face de la lame focalisent les faisceaux diffractés sur le film.

3. Quand $\varphi=\theta+\alpha$ les points $F$ sont répartis à l'intérieur de ce cercle et toutes les courbes se ferment au point $O$.

Les relations (2) et (4) s'annulent en effet pour $\alpha=\theta$.

4. Quand $\varphi=\theta-\alpha$ ou $\alpha-\theta$, les courbes peuvent être assimilées à des droites pour les valeurs de $\theta$ comprises entre $\theta=\alpha$ et $\theta=\alpha \pm 15^{\circ}$.

\section{Applications}

\section{Analyse spectrographique}

Cette méthode de focalisation envisagée dans le cas de la Fig. $3(\alpha<\theta$ avec $\varphi=\theta-\alpha)$ peut être appliquée à l'analyse d'un faisceau hétérogène de rayons $X$ et présente plusieurs avantages:

(1) les lieux de la focalisation sont très voisins de droites quand $\theta$ est voisin de $\alpha$, c'est-à-dire, quand la dispersion est maximum, il est donc possible d'utiliser des plaques photographiques;

(2) on peut obtenir une grande dispersion sous un encombrement réduit: pour les valeurs de $\theta$ voisines de $\alpha$, les valeurs du rapport $O F / l$ sont très grandes;

(3) le trajet total des rayons est court: l'absorption par l'air est donc réduite et dans le cas d'expérience dans le vide les dimensions de l'appareil ne sont pas exagérées;

(4) l'ouverture utile du faisceau incident, et par conséquent l'énergie récupérée, est d'autant plus grande que la distance source-cristal est faible;

(5) comme l'a fait remarquer Tellez-Plasencia, il ne s'agit pas d'une focalisation véritable, puisqu'il n'y a pas concentration simultanée d'énergie; mais les temps de pose se trouvent quand même raccourcis car le cristal étant placé très près de la source, la grande ouverture utile du faisceau incident permet la réflexion simultanée de différentes radiations.

(6) enfin, la silhouette réelle des raies spectrales et le pouvoir séparateur ne sont pas affectés par des aberrations, comme dans les spectrographes à cristal courbe où les zones de focalisation sont des caustiques.

Une première expérience a permis d'obtenir le spectre des radiations caractéristiques du cuivre, en utilisant la face de clivage d'un cristal de calcite. Les raies $K \alpha_{1}$ et $K \alpha_{2}$, distantes de $13 \mathrm{~mm}$. on été obtenues par réflexion sur les plans réticulaires d'intervalle $d=0,805 \AA$.

\section{Images radiographiques de lames cristallines}

Le cas de la transmission $(\alpha>\theta, \varphi=\alpha \pm \theta)$ où l'on trouve un foyer virtuel, présente les meilleures conditions pour obtenir des images agrandies de lames cristallines, le faisceau incident de rayons monochromatiques issus d'une source ponctuelle de rayons $X$ étant réfléchi sélectivement sur une famille de plans réticulaires. Quelques photographies de lamelles de chlorure de sodium ont pu être obtenues dans ces conditions, avec la radiation $K \alpha$ du molybdène.

\section{Bibliographie}

BragG, W. H. \& BragG, W. L. (1914). Proc. Roy. Soc., $A, 88,428$.

Broglie, M. DE (1914). J. Phys. Radium, 4, 101.

Tellez-Plasencia, H. (1943). Arch. Sci. phys. nat. 25, 35. 